\title{
Estabilidade oxidativa da carne caprina armazenada sob congelamento
}

\section{Oxidative stability of meat goats stored under freeze}

\author{
Maria Cecília Oliveira Lima ${ }^{1}$, Luzimar Gonzaga Fernandez ${ }^{2}$, Julliana Izabelle Simionato ${ }^{3}$, Jussimara Barros de Oliveira ${ }^{4}$, \\ Marcondes Viana da Silva ${ }^{5 *}$
}

Resumo: A oxidação lipídica é um dos fatores mais importantes que limitam a vida útil e a estabilidade comercial da carne e produtos cárneos. A oxidação lipídica da carne caprina armazenada sob congelamento, nos tempos de 0, 30, 60 e 120 dias, foi estudada utilizando-se peças do músculo longissimus dorsi, proveniente de animais caprinos, da raça Anglo-Nubiana, machos, não castrados, com idade inferior a oito meses, criados em regime extensivo. Para tanto, realizou-se análise do índice de substâncias reativas ao ácido 2-tiobarbitúrico (TBARS), do índice de peróxido e a análise cromatográfica de fase gasosa. De acordo com os resultados encontrados para o TBARS e o índice de peróxido, as amostras não variaram de forma significativa ( $p>0,05)$ em relação ao tempo de estocagem. Destacaram-se como ácidos graxos majoritários, os ácidos palmítico, esteárico e oléico. A temperatura de armazenamento de $-18{ }^{\circ} \mathrm{C}$ contribuiu para a manutenção da qualidade da carne caprina por, pelo menos, 120 dias.

Palavras-Chave: malonaldeído, TBARS, qualidade da carne, lipoperoxidação, ácidos graxos

Abstract: Lipid oxidation is one of the most important factors that limit the useful life and the commercial stability of meat and meat products. Lipid oxidation of goat meat stored under freezing, at times 0, 30, 60 and 120 days, was studied using parts of the longissimus dorsi muscle, from animals goats, Anglo-Nubian breed, male, unneutered, with less than eight months old raised extensively. To do so, we performed analysis of index to 2-thiobarbituric reactive substances (TBARS) acid, peroxide value and the chromatographic analysis of gas phase substances. According to the results found for TBARS and peroxide, the samples did not differ significantly $(\mathrm{p}>0.05)$ in relation to storage time. Stood out as major fatty acids, palmitic, stearic and oleic. The storage temperature of $-18{ }^{\circ} \mathrm{C}$ contributed to the maintenance of the quality of goat meat for at least 120 days.

Key words: malondialdehyde, TBARS, meat quality, lipid peroxidation, fatty acids

\footnotetext{
*Autor para correspondência

Recebido para publicação em 12/08/2014; aprovado em 20/04/2015

${ }^{1}$ Mestre em Engenharia de Alimentos, Vigilância Sanitária Municipal de Itapetinga, CEP 45.700-000, Itapetinga-Ba. E-mail: cissaluiza@ hotmail.com

${ }^{2}$ Professora D. Sc. da Universidade Federal da Bahia, CEP 40.110-100, Salvador-BA. E-mail: luzimar@ucsal.br

${ }^{3}$ Professora D. Sc. da Universidade Tecnológica Federal do Paraná - UFTPR, CEP 86.036-360, Londrina-PR. E-mail: jusimionato@ gmail.com

${ }^{4}$ Mestre em Engenharia de Alimentos, Núcleo de Estudos em Ciência de Alimentos - UESB, Universidade Estadual do Sudoeste da Bahia, CEP 45.700-000,

Itapetinga-Ba. E-mail: marah_disc@hotmail.com

5 Professor D. Sc. da Universidade Estadual do Sudoeste da Bahia - UESB, Pça. Primavera, 40, Primavera, CEP 45.700-000, Itapetinga-Ba. E-mail: mviana@hotmail.com
} 


\section{INTRODUÇÃO}

Os caprinos são animais de pequeno porte, apresentam a carcaça pequena, magra e pouco compacta, porém aumenta e torna-se compacta à medida que ganham peso (MADRUGA, 1999). As carcaças caprinas são normalmente pequenas, com menos gordura do que as carcaças de ovinos de idades e sexos comparáveis (WEBB et al., 2005).

A carne caprina é considerada um produto com alto potencial de expansão em decorrência da sua composição química. Quando comparada a outras carnes vermelhas apresenta quantidades semelhantes em proteína e ferro, entretanto, quantidades menores de gorduras totais (MALAN, 2000).

A gordura é um fator determinante da qualidade das carcaças que influencia as propriedades organolépticas, a palatabilidade e o valor nutricional. Além disso, a carne caprina é rica em ácidos graxos insaturados, sendo estes benéficos a saúde humana, contribuindo para a redução do risco de obesidade, doenças cardiovasculares, dentre outras (WEBB et al., 2005; CASEY, 1992).

A composição química da carne caprina, e mais especificamente da gordura intramuscular, é influenciada por fatores como peso vivo no abate, genótipo, músculo, sexo e dieta. Em bovinos e ovinos, a dieta tem um grande impacto sobre a deposição da gordura intramuscular, bem como sobre a concentração de ácidos graxos saturados e ácidos graxos polinsaturados (DE SMET et al., 2004).

Em comparação à carne bovina, suína e ovina, a carne caprina apresenta uma fração de lipídios menor, menos saturada e com um teor de colesterol reduzido (PINKERTON \& McMILLIN, 1997; FRANCO, 2003). A gordura saturada da carne caprina é $40 \%$ menor que a de frango sem pele, $850 \%$ menor que a de bovinos, $1100 \%$ que a de suínos e 900\% que a de ovinos. Isso a torna um alimento de grande interesse para a composição de uma dieta adequada, principalmente quando se leva em consideração o elevado índice de problemas cardiovasculares na população (PINKERTON \& McMILLIN, 1997).

A carne caprina possui destaque nas concentrações de ácidos graxos poliinsaturados, principalmente os ômega-3 e ômega-6 e nos elevados níveis de ácidos graxos insaturados (MADRUGA, 2004).

Os caprinos são animais que depositam mais gordura abdominal e menos gordura subcutânea e intramuscular (COLOMER-ROCHER et al., 1992). Apresentam uma relação de AGP/AGS (ácidos graxos poliinsaturados/ácidos graxos saturados) maior, quando comparado a ovinos e bovinos (BANSKALIEVA et al., 2000). Estas características estão de acordo ao preconizado pela Organização Mundial de Saúde cuja recomendação é a substituição na dieta de AGS (ácidos graxos saturados) por AGM (ácidos graxos monoinsaturados) e/ou AGP (ácidos graxos poliinsaturados) para prevenir doenças cardíacas e alguns distúrbios inflamatórios (ENSER et al., 1998; HAGLUND et al., 1998).

A oxidação lípidica em carnes e seus derivados é influenciada por diversos fatores, destacando-se a composição de ácidos graxos, principalmente os poliinsaturados que são mais suscetíveis à oxidação, o teor de vitamine $\mathrm{E}(\alpha$ tocoferol), bem como de prooxidantes, tais como o ferro encontrado livre nos músculos. Esse processo causa o desenvolvimento de sabores indesejáveis, perda de cor, produção de substâncias potencialmente tóxicas, como o malonaldeído e óxidos de colesterol, e também perda do valor nutricional não só da fração lipídica, mas também, de pigmentos, proteínas, carboidratos e de vitaminas (LEGOYNIE et al., 2012; GRAY et al., 1996).

Os produtos secundários da lipoperoxidação têm efeitos biológicos nocivos e a saúde e têm sido relacionados com a etiologia de doenças neurodegenerativas e cardiovasculares, bem como o desenvolvimento de diferentes tipos de câncer e processos mutagênicos (NIKI, 2014; SPICKETT, 2013; SCHROEPFER, 2002).

Dentro dos sistemas de conservação de alimentos, o uso de baixas temperaturas tem sido generalizado, especialmente para produtos altamente perecíveis, como a carne. O congelamento é um método de conservação comumente utilizado para armazenar carnes por períodos relativamente longos de tempo (PIETRASIK \& JANZ, 2008).

No Brasil, a utilização do congelamento de carnes é uma prática habitual que visa atender o processo de distribuição interestadual e exportações de carnes (PARDI et al., 2006). O congelamento da carne tem sido bastante utilizado por ser o método de conservação que menos deprecia as qualidades sensoriais, químicas e nutritivas do produto final (LAWRIE, 2005). Porém, alterações como a desidratação, rancidez oxidativa e perdas de suco, são efeitos negativos que podem ser observados em carnes devido ao processo de congelamento (MONTEIRO et al., 2002; CAMPAÑONE et al., 2006).

Assim sendo, torna-se imperativo a utilização de métodos de conservação, tais como o congelamento, não só visando preservar o valor nutricional dos alimentos, mas também para minimizar a oxidação lípidica, e assim, atender a exigência dos consumidores que buscam alimentos saudáveis.

Objetivou-se com o presente estudo, determinar a composição de ácidos graxos da fração lipídica e utilizar o índice de substâncias reativas ao ácido 2-tiobarbitúrico (TBARS) e o índice de peróxido como marcadores bioquímicos da lipoperoxidação do músculo longissimus dorsi da carne caprina armazenada sob congelamento.

\section{MATERIAL E MÉTODOS}

O experimento foi conduzido no Laboratório do Núcleo de Estudos em Ciências de Alimentos (NECAL) da Universidade Estadual do Sudoeste da Bahia (UESB), Campus de Itapetinga-BA.

Foram obtidos 28 pares de peças do músculo longíssimos dorsi, proveniente de 28 machos caprinos, da raça Anglo-Nubiana, não castrados, com idade inferior a oito meses, observando o sistema de tipificação de carcaças caprino, cedidos pela Unidade Experimental de Caprinos e Ovinos (UECO) da UESB.

As peças foram acondicionadas em sacos plásticos hermeticamente fechados, aos pares, codificadas e armazenadas $\left(-18^{\circ} \mathrm{C}\right)$ até o momento das análises. Utilizou-se sete amostras como controle (tempo zero), sete amostras após 30 dias de armazenamento, sete amostras após 60 dias e sete amostras após 120 dias estocadas sob congelamento, sendo todas as amostras escolhidas ao acaso. 
As amostras controle foram analisadas frescas. Cada animal foi considerado uma unidade experimental, constituindo assim, uma repetição, totalizando sete repetições por período. Todas as análises foram realizadas em triplicata.

\section{Determinações químicas}

\section{Índice de substâncias reativas ao ácido 2-tiobarbitúrico -} TBARS

As análises das substâncias reativas ao ácido 2tiobarbitúrico (TBARS) foram determinadas utilizando o método de destilação, conforme o protocolo recomendado por Tarladgis et al. (1960), com adaptações. O teste baseia-se na reação entre o ácido 2-tiobarbitúrico com o malonaldeído (MAD), produzindo um composto de cor vermelha, medido espectrofotometricamente a $532 \mathrm{~nm}$. Os resultados foram expressos em mg de malonaldeído por $\mathrm{kg}$ de amostra.

As curvas analíticas foram construídas através de diluições apropriadas da solução padrão de 1,1,3,3tetrametoxipropano (TEP, Sigma Chemical Co.) na concentração de $1.10^{-3} \mathrm{M}$, de modo a obter quantidades que variam entre $1.10^{-8}$ a $8.10^{-8}$ moles de malonaldeído por $3 \mathrm{~mL}$. O espectro de absorção foi realizado com uma solução de $2.10^{-7}$ mols de malonaldeído.

O percentual de recuperação foi calculado segundo Sinnhuber \& Yu (1958), sendo calculado a partir do padrão, conforme equação 1 .

$$
\text { IP } \%=\frac{\left[A_{2}\right]-\left[A_{1}\right]}{\left[A_{2}\right]}
$$

\section{Em que:}

$\mathrm{P}=$ percentual de recuperação; $\mathrm{A}_{2}=$ absorbância da amostra destilada contendo TEP; $\mathrm{A}_{1}=$ absorbância da amostra.

O valor de " $k$ " foi calculado a partir da curva analítica linear a partir de diluições realizadas conforme equação 2 .

$$
\mathrm{K}_{\text {destilação }}=\frac{S}{\mathrm{~A}} \times M M-M A D \times \frac{10^{7}}{C} \times \frac{100}{P}
$$

Onde:

$\mathrm{S}=$ concentração molar do padrão $\left(1.10^{-8} \mathrm{~mol}\right.$ de MDA. $\left.3 \mathrm{~mL}^{-1}\right) ; \mathrm{A}=$ absorbância do padrão;

MM-MAD = massa molar do malonaldeído $=$ 72,063; $\mathrm{C}=$ peso da amostra em gramas; $\mathrm{P}=$ porcentagem de recuperação.

\section{Índice de peróxido - IP}

A determinação do índice de peróxido foi realizada de acordo com a metodologia proposta pela AOCS (2003). Para o cálculo do índice de peróxido (mEq de peróxidos. 1000 $\mathrm{g}^{-1}$ de amostra) foi utilizada a equação 3 .

$$
\begin{aligned}
& \mathrm{IP}=\left(m \text { Eq de peróxidos } .100 \mathrm{~g}^{-1} \text { de amostra }\right)=\frac{\mathrm{S} \times \mathrm{N} \times 1000}{g \text { amostra }}(\text { Eq. } 3) \\
& \text { Em que: } \\
& \mathrm{S}=\text { volume de } \mathrm{Na}_{2} \mathrm{~S}_{2} \mathrm{O}_{3} \text { gasto na titulação da } \\
& \text { amostra; } \mathrm{N}=\text { normalidade da solução de } \mathrm{Na}_{2} \mathrm{~S}_{2} \mathrm{O}_{3} .
\end{aligned}
$$

\section{Composição de ácidos graxos por cromatografia gasosa}

A transesterificação dos ácidos graxos extraídos foi realizada pelo Método ISO 5509 (ISO, 1978). Os ésteres metílicos foram identificados e quantificados em um cromatógrafo de fase gasosa (Thermo-Finnigan) utilizando uma coluna capilar de sílica fundida BPX-70 (120m x $0,25 \mathrm{~mm}$ d.i). A temperatura do injetor e do detector de ionização de chama (FID) foram $250^{\circ} \mathrm{C}$ e $280^{\circ} \mathrm{C}$ respectivamente. A temperatura da coluna foi programada a $140^{\circ} \mathrm{C}$ por 10 minutos, seguido por uma primeira rampa de $15^{\circ} \mathrm{C} \mathrm{min}^{-1}$ até atingir $200^{\circ} \mathrm{C}$ por 1 minuto. A segunda rampa foi de $10^{\circ} \mathrm{C} \min ^{-1}$ até atingir $230^{\circ} \mathrm{C}$ por 1 minuto. A terceira rampa $0,4^{\circ} \mathrm{C} \mathrm{min}{ }^{-1}$ até atingir $233^{\circ} \mathrm{C}$ por 3 minutos. A quarta rampa $0,5^{\circ} \mathrm{C} \min ^{-1}$ até atingir $238^{\circ} \mathrm{C}$ por 2 minutos. $\mathrm{O}$ tempo total de análise foi de 55 minutos.

As vazões dos gases (White Martins) foram de 30 $\mathrm{mL} \min ^{-1}$ para o gás hidrogênio, $30 \mathrm{~mL} \mathrm{~min}^{-1}$ para o nitrogênio e $250 \mathrm{~mL} \mathrm{~min}^{-1}$ para o ar sintético. Os volumes de injeção foram de $1,2 \mu \mathrm{L}$ e as injeções foram realizadas manualmente em triplicata. As áreas dos picos dos ésteres metílicos de ácidos graxos foram determinadas através do software ChromQuest 4.1.

Para a identificação dos ácidos graxos, foram comparados os tempos de retenção e os picos das amostras com a do padrão que correspondia a uma mistura de 37 padrões de ésteres metílicos de ácidos graxos (189-19 Sigma, EUA).

\section{Análise estatística}

O experimento foi conduzido em delineamento inteiramente casualizado. Os resultados foram apresentados como média \pm desvio padrão $(\mathrm{DP})$ e submetidos à análise de variância (ANOVA) com aplicação do Teste de Tukey, ao nível de significância de 5\%, usando o Sistema de Análises Estatísticas e Genética (SAEG) versão 8.0.

\section{RESULTADOS E DISCUSSÃO}

\section{Estabilidade oxidativa}

Os resultados obtidos para o índice de peróxido (IP) da carne caprina para os quatro tempos de estocagem $(0 ; 30$; 60 e 120 dias) estão apresentados na Tabela 1 . Os índices de peróxidos não apresentaram diferenças significativas $(p>0,05)$ para os diferentes tempos de congelamento variando entre 0,38 a $0,53 \mathrm{mEq} \cdot 1000 \mathrm{~g}^{-1}$. Os resultados observados neste estudo encontram-se em conformidade com a legislação brasileira, cujo valor permitido é estimado em $1 \mathrm{mEq} .1000 \mathrm{~g}^{-1}$ para as carnes mecanicamente separada de aves, bovinos e suínos (BRASIL, 2000).

Os resultados obtidos para o TBARS encontram-se apresentados na Tabela 2. Os teores médios variaram de 0,07 a 0,22 mg.MDA. $\mathrm{kg}^{-1}$. Observou-se que não houve influência significativa $(\mathrm{p}>0,05)$ do período de armazenamento sobre o índice de TBARS do músculo longissimus dorsis da carne caprina analisada. O processo de deterioração oxidativa manteve-se sob controle ao longo dos 120 dias de armazenamento, não influenciando desta forma a qualidade da carne. 
Tabela 1. Determinação do índice de peróxido $\left(\mathrm{mEq} \cdot 1000 \mathrm{~g}^{-1}\right)$ da carne caprina armazenada a $-18^{\circ} \mathrm{C}$

\begin{tabular}{|c|c|c|c|c|}
\hline \multicolumn{5}{|c|}{ Tempo (dias) } \\
\hline Amostra & $\mathbf{0}$ & 30 & 60 & 120 \\
\hline 1 & 0,24 & 0,44 & 0,15 & 0,65 \\
\hline 2 & 0,65 & 0,75 & 0,26 & 0,52 \\
\hline 3 & 0,25 & 0,45 & 0,45 & 0,33 \\
\hline 4 & 0,54 & 0,23 & 0,65 & 0,75 \\
\hline 5 & 0,25 & 0,62 & 0,24 & 0,44 \\
\hline 6 & 0,95 & 0,35 & 0,53 & 0,85 \\
\hline 7 & 0,35 & 0,14 & 0,38 & 0,22 \\
\hline Média & $0,46^{\mathrm{A}}$ & $0,42^{\mathrm{A}}$ & $0,38^{\mathrm{A}}$ & $0,53^{\mathrm{A}}$ \\
\hline $\mathrm{CV}(\%)$ & 49,79 & & & \\
\hline
\end{tabular}

Pereira et al. (2006) encontraram diferença significativa para a carne de ema armazenada sob diferentes períodos de congelamento $(0 ; 15 ; 30$ e 60 dias $)$ a $-18^{\circ} \mathrm{C}$, onde houve um aumento no período de 0 a 60 dias. Stika et al. (2007) observaram um aumento nos valores de TBARS da carne bovina com o aumento do tempo de estocagem sob congelamento a $-29^{\circ} \mathrm{C}$.
Valores de TBARS superiores a $1 \mathrm{mg}$. MDA. $\mathrm{kg}^{-1}$ são perceptíveis, resultando em off-flavours (DJENANE et al., 2002). O percentual de recuperação do MAD obtido neste experimento foi de $82,71 \%$, superior ao encontrado por Tarladgis et al. (1960) quando descreveram a aplicação do método de destilação para quantificação do MDA em carnes.

Tabela 2. Determinação do TBARS (mg. MDA.kg $\left.{ }^{-1}\right)$ da carne caprina armazenada a $-18^{\circ} \mathrm{C}$

\begin{tabular}{ccccc}
\hline Amostra & $\mathbf{0}$ & Tempo (dias) & $\mathbf{6 0}$ & $\mathbf{1 2 0}$ \\
\hline 1 & 0,08 & 0,16 & 0,44 & 0,12 \\
2 & 0,06 & 0,08 & 0,17 & 0,04 \\
3 & 0,03 & 0,13 & 0,11 & 0,29 \\
5 & 0,29 & 0,29 & 0,12 & 0,26 \\
6 & 0,04 & 0,09 & 0,12 & 0,83 \\
7 & 0,03 & 0,12 & 0,03 \\
\hline Média & 0,02 & $0,14^{\mathrm{A}}$ & $0,22^{\mathrm{A}}$ & $0,22^{\mathrm{A}}$ \\
\hline
\end{tabular}

\footnotetext{
${ }^{\mathrm{A}, \mathrm{B}}$ letras maiúsculas distintas nas colunas, indicam valores que diferem estatisticamente entre si $(\mathrm{p}<0,05)$ pelo teste de Tukey a $5 \%$ de probabilidade
}

Testes como TBARS e índice de peróxido são utilizados no controle de qualidade de óleos, gorduras e produtos que os contenham, por fornecerem informações a respeito do estado oxidativo, na predição da rancidez do alimento analisado. A rancidez é uma das deteriorações mais importantes que ocorrem nesses produtos, definindo a vida útil, na medida que gera produtos indesejáveis sensorialmente e oxida vitaminas lipossolúveis e ácidos graxos essenciais reduzindo o valor nutricional dos alimentos gordurosos (CECCHI, 1999).

\section{Perfil de ácidos graxos}

A composição de ácidos graxos da carne caprina armazenada a $-18^{\circ} \mathrm{C}$ encontra-se apresentada na Tabela 3. Observou-se 22 ácidos graxos, sendo que, dos ácidos identificados oito foram ácidos graxos saturados, seis ácidos graxos monoinsaturados e oito ácidos graxos poliinsaturados. A proporção de ácidos graxos saturados em relação ao total de ácidos quantificados representou 59,5\%, enquanto que os ácidos graxos insaturados contribuíram com 40,5\%. Os ácidos graxos foram analisados nos tempos 0,30 e 120 dias de armazenamento.

Os ácidos graxos de maiores proporções encontrados nos lipídios do logissimos dorsi da carne caprina foram o palmítico e o esteárico (ácidos graxos saturados), o oléico (ácido graxo monoinsaturado) e o linoléico (ácido graxo poliinsaturado). Destes ácidos graxos majoritários, apenas o ácido palmítico não apresentou diferença significativa $(\mathrm{p}>0,05)$ nos tempos de armazenamento a $-18^{\circ} \mathrm{C}$.

A razão entre ácidos graxos poliisaturados e saturados (AGP/AGPS) representa uma ferramenta que tem sido utilizada para a avaliação da qualidade da gordura ingerida pelos consumidores. O Department of Healt UK recomenda que a relação AGP/AGPS dos alimentos seja superior a 0,4 visando à prevenção de doenças cardiovasculares (DH, 1994). Essa relação é utilizada para calcular o fator de risco dos alimentos, considerando a elevação do colesterol. 
Tabela 3. Composição de ácidos graxos em mg. $\mathrm{g}^{-1}$ da carne caprina armazenada a $-18^{\circ} \mathrm{C}$

\begin{tabular}{|c|c|c|c|c|c|}
\hline \multirow[b]{2}{*}{ Ácidos Graxos } & \multicolumn{4}{|c|}{ Tempo de armazenamento (dias) } & \multirow[t]{2}{*}{ CV $(\%)$} \\
\hline & & $\mathbf{0}$ & 30 & 120 & \\
\hline \multicolumn{6}{|l|}{ Saturados } \\
\hline Mirístico & $14: 0$ & $1,88^{\mathrm{A}} \pm 0,38$ & $2,62^{\mathrm{A}} \pm 0,58$ & $2,58^{\mathrm{A}} \pm 0,51$ & 30,04 \\
\hline Pentadecanóico & $15: 0$ & $0,49^{\mathrm{A}} \pm 0,09$ & $0,48^{\mathrm{A}} \pm 0,03$ & $0,47^{\mathrm{A}} \pm 0,07$ & 20,33 \\
\hline Palmítico & $16: 0$ & $25,09^{\mathrm{A}} \pm 2,49$ & $24,19^{\mathrm{A}} \pm 1,09$ & $21,97^{\mathrm{A}} \pm 2,33$ & 11,58 \\
\hline Margárico & 17:0 & $1,46^{\mathrm{A}} \pm 0,31$ & $1,55^{\mathrm{A}} \pm 0,22$ & $1,42^{\mathrm{A}} \pm 0,17$ & 22,40 \\
\hline Esteárico & 18:0 & $31,03^{A} \pm 5,88$ & $19,48^{\mathrm{B}} \pm 0,73$ & $17,86^{\mathrm{B}} \pm 0,85$ & 19,72 \\
\hline Araquídico & 20:0 & $0,18^{\mathrm{C}} \pm 0,05$ & $0,48^{\mathrm{B}} \pm 0,13$ & $1,00^{\mathrm{A}} \pm 0,11$ & 24,83 \\
\hline Heneicosanóico & 21:0 & $0,38^{\mathrm{B}} \pm 0,09$ & $0,80^{\mathrm{B}} \pm 0,30$ & $1,83^{\mathrm{A}} \pm 0,36$ & 35,21 \\
\hline Behênico & $22: 0$ & $1,04^{\mathrm{C}} \pm 0,40$ & $3,56^{\mathrm{B}} \pm 1,33$ & $9,37^{\mathrm{A}} \pm 1,82$ & 37,77 \\
\hline \multicolumn{6}{|l|}{ Monoinsaturados } \\
\hline Miristoléico & $14: 1$ & $0,15^{\mathrm{A}} \pm 0,03$ & $0,14^{\mathrm{A}} \pm 0,03$ & $0,15^{\mathrm{A}} \pm 0,03$ & 25,72 \\
\hline Pentadecenóico & $15: 1$ & $0,20^{\mathrm{A}} \pm 0,02$ & $0,20^{\mathrm{A}} \pm 0,02$ & $0,21^{\mathrm{A}} \pm 0,03$ & 15,68 \\
\hline Palmitoléico & $16: 1$ & $1,15^{\mathrm{A}} \pm 0,26$ & $1,41^{\mathrm{A}} \pm 0,15$ & $0,43^{\mathrm{B}} \pm 0,06$ & 22,95 \\
\hline Heptadecenóico & $17: 1$ & $0,73^{\mathrm{B}} \pm 0,22$ & $1,11^{\mathrm{A}} \pm 0,10$ & $1,11^{\mathrm{A}} \pm 0,05$ & 19,89 \\
\hline Vaccênico & $18: 1 n-7 t$ & $1,31^{\mathrm{A}} \pm 0,12$ & $1,34^{\mathrm{A}} \pm 0,27$ & $0,75^{\mathrm{B}} \pm 0,14$ & 25,04 \\
\hline Oléico & $18: 1 n-9 c$ & $35,31^{\mathrm{A}} \pm 3,69$ & $31,66^{\mathrm{A}} \pm 3,90$ & $23,98^{\mathrm{B}} \pm 2,44$ & 14,67 \\
\hline \multicolumn{6}{|l|}{ Poliinsaturados } \\
\hline Linolênico & $18: 2 n-6$ & $2,17^{\mathrm{B}} \pm 0,37$ & $2,87^{\mathrm{B}} \pm 0,55$ & $9,45^{\mathrm{A}} \pm 1,17$ & 24,61 \\
\hline CLA & CLAc9,t11 & $0,15^{\mathrm{B}} \pm 0,02$ & $0,19^{\mathrm{B}} \pm 0,06$ & $0,27^{\mathrm{A}} \pm 0,04$ & 26,71 \\
\hline Alfa linolênico & $18: 3 n-3$ & $0,55^{\mathrm{A}} \pm 0,19$ & $0,33^{\mathrm{B}} \pm 0,04$ & $1,14^{\mathrm{B}} \pm 0,02$ & 41,66 \\
\hline Gama linolênico & $18: 3 n-6$ & $0,22^{\mathrm{A}} \pm 0,02$ & $0,16^{\mathrm{A}} \pm 0,03$ & $0,21^{\mathrm{A}} \pm 0,04$ & 25,58 \\
\hline Docosadienóico & $22: 2 n-6$ & $0,34^{\mathrm{B}} \pm 0,13$ & $0,59^{\mathrm{B}} \pm 0,19$ & $2,84^{\mathrm{A}} \pm 0,47$ & 34,04 \\
\hline Timnodônico & $20: 5 n-3$ & $0,15^{\mathrm{B}} \pm 0,06$ & $0,14^{\mathrm{B}} \pm 0,06$ & $0,45^{\mathrm{A}} \pm 0,07$ & 35,14 \\
\hline Clupanodônico & $22: 5 n-3$ & $0,53^{\mathrm{B}} \pm 0,14$ & $1,03^{\mathrm{B}} \pm 0,34$ & $4,12^{\mathrm{A}} \pm 0,64$ & 31,96 \\
\hline Cervônico & $22: 6 n-3$ & $0,25^{\mathrm{A}} \pm 0,08$ & $0,10^{\mathrm{B}} \pm 0,03$ & $0,06^{\mathrm{B}} \pm 0,01$ & 48,95 \\
\hline
\end{tabular}

${ }^{\mathrm{A}, \mathrm{B}}$ letras maiúsculas distintas nas colunas, indicam valores que diferem estatisticamente entre si (p<0,05) pelo teste de Tukey a $5 \%$ de probabilidade

A relação entre AGPI/AGS apresentou uma variação de 0,07 a $0,32 \mathrm{mg} \cdot \mathrm{g}^{-1}$ (Tabela 4). Estes resultados foram menores que o limite supracitado, o que implica em um desequilíbrio na ingestão de ácidos graxos. Resultados semelhantes (AGPI/AGS) foram verificados por Banskalieva et al. (2000) quando analisaram a composição de ácidos graxos da carne caprina $(0,16$ a 0,49$)$, cujos valores variaram dependentes da raça, do músculo e da idade do animal. No entanto, Zapata et al. (2003), analisando esta mesma relação reportaram na carne caprina variação menor $(0,9$ a 1,4$)$.

Tabela 4. $\sum$ ácidos graxos saturados, mono e insaturados, AGPI/AGS, $\omega-6, \omega-3$ e $\omega-6 / \omega-3$ em mg.g ${ }^{-1}$ para carne caprina armazenada a $-18^{\circ} \mathrm{C}$

\begin{tabular}{lcccc}
\hline Ácidos Graxos & \multicolumn{3}{c}{ Tempo de armazenamento (Dias) } & CV (\%) \\
\cline { 2 - 4 } & $\mathbf{0}$ & $\mathbf{3 0}$ & $\mathbf{1 2 0}$ & 10,32 \\
$\mathrm{AGS}^{1}$ & $60,12^{\mathrm{A}} \pm 6,17$ & $51,64^{\mathrm{B}} \pm 1,94$ & $55,10^{\mathrm{AB}} \pm 2,18$ & 13,80 \\
$\mathrm{AGM}^{2}$ & $38,88^{\mathrm{A}} \pm 3,61$ & $35,88^{\mathrm{A}} \pm 3,61$ & $26,63^{\mathrm{B}} \pm 2,18$ & 24,48 \\
$\mathrm{AGPI}^{3}$ & $4,39^{\mathrm{B}} \pm 0,59$ & $5,44^{\mathrm{B}} \pm 1,05$ & $17,57^{\mathrm{A}} \pm 2,10$ & 27,09 \\
$\mathrm{AGPI}^{\mathrm{A} A G S}$ & $0,07^{\mathrm{B}} \pm 0,02$ & $0,10^{\mathrm{B}} \pm 0,02$ & $0,32^{\mathrm{A}} \pm 0,04$ & 24,92 \\
$\sum \omega-6^{5}$ & $2,74^{\mathrm{B}} \pm 0,42$ & $3,62^{\mathrm{B}} \pm 0,64$ & $12,50^{\mathrm{A}} \pm 1,48$ & 26,59 \\
$\sum \omega-3^{6}$ & $1,49^{\mathrm{B}} \pm 0,34$ & $1,62^{\mathrm{B}} \pm 0,35$ & $4,79^{\mathrm{A}} \pm 0,60$ & 19,74 \\
$\omega-6 / \omega-3^{7}$ & $1,97^{\mathrm{A}} \pm 0,44$ & $2,31^{\mathrm{A}} \pm 0,28$ & $2,61^{\mathrm{A}} \pm 0,12$ & \\
\hline
\end{tabular}

${ }^{1}$ Somatório de Ácidos Graxos Saturados (14:0, 15:0, 16:0, 17:0, 18:0, 20:0, 21:0 e 22:0);

${ }^{2}$ Somatório de Ácidos Graxos Monoinsaturados (14:1, 15:1, 16:1, 17:1, 18:1n-7t e 18:1n-9c);

${ }^{3}$ Somatório de Ácidos Graxos Poliinsaturados (18:2n-6, CLAc9t11, CLAt10c12, 18:3n-3, 20:3n-3 e 20:3n-6);

${ }^{4}$ Razão entre os Ácidos Graxos Poliinsaturados e Saturados;

${ }^{5}$ Somatório do $\omega-6$ (18:2n-6, 18:3n-6 e 22:2n-6);

${ }^{6}$ Somatório do $\omega-3$ (18:3n-3, 20:5n-3, 22:5n-3 e 22:6n-3);

${ }^{7}$ Razão entre $\omega-6 / \omega-3$;

Diversos estudos disponíveis na literatura têm relatado os efeitos dos óleos e gorduras na nutrição humana, bem como a relação adequada de ácidos graxos das séries ômega-6 e ômega-3 (YU et al., 2013; CANDELA et al., 2011; SIMOPOULOS, 2008). Neste íterim, o Departamento de Saúde da Inglaterra recomenda que o valor da razão $\omega-6 / \omega-3$ não seja superior a 4,0 (DH, 1994).

A razão $\omega-6 / \omega-3$ nas amostras da carne caprina analisadas não apresentou diferença significativa $(p>0,05)$ ao longo do período de armazenamento a $-18^{\circ} \mathrm{C}$. Os valores encontrados variaram entre 1,97 a 2,61 e não excedeu o limite recomendado, o que representa um fator importante para a saúde humana.

Os ácidos graxos ômega-6 e ômega-3 desempenham funções biológicas opostas, competindo entre si pelo mesmo sistema enzimático nas reações de dessaturação e alongamento de cadeia (EMKEN et al., 1994). 
O metabolismo do ácido alfa-linolênico (ômega-3) resulta nos ácidos eicosapenatanóico (EPA) e decosaehexanóico (DHA), sendo estes, precursores dos mediadores químicos prostaglandinas e leucotrienos, relacionados potencialmente com efeitos antitrombóticos, antiateroscleróticos, antiinflamatórios e agem na melhora da função endotelial e na diminuição da pressão arterial e concentração de triglicerídeos (HARRIS et al., 2006; DIN et al., 2004; STTOL, 2002)

Em contraste, o ácido linoléico (ômega-6) é metabolizado em ácido araquidônico, precursor da síntese dos eicosanóides, relacionados com os processos de infecção, inflamação, lesão tecidual, modulação do sistema imune e agregação plaquetária e dentre outras (SAADATIAN-ELAHI, 2004).

\section{CONCLUSÕES}

De acordo com os resultados obtidos para o índice de peróxido e de substâncias reativas ao ácido 2-tiobarbitúrico, marcadores bioquímicos da lipoperoxidação, sugere-se ser possível o armazenamento da carne caprina por até 120 dias a $-18^{\circ} \mathrm{C}$.

Quanto ao perfil dos ácidos graxos, constatam-se como ácidos graxos majoritários o palmítico, o esteárico e oléico. Destes ácidos graxos, o conteúdo do palmítico manteve-se constante, no entanto, o ácido esteárico e o oléico apresentaram redução em seus teores.

\section{AGRADECIMENTOS}

Os autores agradecem à Unidade Experimental de Caprinos e Ovinos (UECO) da UESB pelo fornecimento da matéria-prima objeto desse estudo.

\section{REFERÊNCIAS BIBLIOGRÁFICAS}

AOCS. Official Methods and Recommended Practices of the American Oil Chemists' Society. 4. Champaign: AOCS Press, 2003.

BANSKALIEVA, V.; SAHLU, T. \& GOETSCH, A. L. Fatty acid composition of goat muscles and fat depots: a review. Small Ruminant Research, v.37, n. 2, 255-268 p, 2000 .

BRASIL. Ministério da Agricultura - Secretaria da Defesa Agropecuária. Instrução Normativa $\mathrm{N}^{\circ} 4$, de 31 de março de 2000. Regulamento Técnico Para Fixação de Identidade e Qualidade de Carne Mecanicamente Separada (Cms) de Aves, Bovinos e Suínos. Diário Oficial [da] República Federativa do Brasil, Brasília, publicado em 05 de abr. de 2000.

CANDELA, C. G.; L. LÓPEZ, M. B. \& KOHEN, V. L. Importance of a balanced omega 6/omega 3 ratio for the maintenanceof health. Nutritional recommendations. Nutrición Hospitalaria, v. 26, n. 2, p.323-329, 2011.

CAMPAÑONE, L. A.; ROCHE, L. A.; SALVADOR, V. O. \& MASCHERONI, R. H. Structural studies on unpackaged foods during their freezing and storage. Journal Food Science, v. 71, n. 5, p. E218-E226, 2006.
CASEY, N. H. Goat meat in human nutrition. Proceedings V International Conference on Goats. Indian Council of Agricultural Research, New Delhi, v. 2, n. 2, p. 581-598, 1992.

CECCHI, H. M. Fundamentos Teóricos e Práticos em Análise de Alimentos, Campinas: Editora da Unicamp. Coleção Livro Texto, 1999. 119p.

CHRISTIE, W. W. Lipid analysis. Oxford: Pergamon Press, Chromatographic and spectroscopic analysis of lipids: general principles, cap. 3, p. 25-49, 1982.

COLOMER-ROCHER, F. F.; KIRTON, A. H.; MERCECER, G. J. \& DUGANZICH, D. M. Carcass composition of New Zealand Saanen goats slaughtered at different weights. Small Ruminant Research, v. 7, n. 2, p. 161173, 1992.

DE SMET, S.; RAES, K. \& DEMEYER, D. Meat fatty acid composition as affected by fatness and genetic factors: a review. Animal Research, v. 53, p. 81-98, 2004.

DEPARTMENT OF HEALTH. Nutritional aspects of cardiovascular disease: report of the cardiovascular review group. London: HMSO, 1994.

DIN, J. N.; NEWBY, D. E. \& FLANPAN, A. D. Omega 3 fatty acids and cardiovascular disease-fishing for a natural treatment. British Medical Journal, v. 328, n. 3, p. 30-35, 2004.

DJENANE, D.; SANCHEZ-ESCALANTE A.; BELTRAN, J. A. \& RONCALES, P. Ability of $\alpha$-tocopherol, taurine and rosemary, in combination with vitamin $\mathrm{C}$, to increase the oxidative stability of beef steaks packanged in modified atmospheres. Food Chemistry, v. 76, n. 4, p. 407-415, 2002.

EMKEN, E. A.; ADLOF R. O. \& GULLEY, R. M. Dietary linoleic acid influences desaturation and acylation of deuterium-labeled linoleic and linolenic acids in young adult males. Biochim Biophys Acta, v. 1213, n. 3, p.277-288, 1994.

ENSER, M.; HALLET, K. G.; HEWETT, B.; FURSEY, G. A.J.; WOOD, J. D. \& HARRINGTON, G. Fatty acid content and composition of UK beef and lamb muscle in relation to production system and implications for human nutrition. Meat Science, v. 49, n. 3, p. 329-341, 1998.

FRANCO, G. Tabela de Composição Química dos Alimentos. 9 ed., São Paulo: Editora Atheneu, 2003, $307 \mathrm{p}$.

GRAY, J. I., GOMAA, E. A. \& BUCKLEY, D. J. Oxidative quality and shelf life of meats. Meat Science, v. 43, n. 1, p. S111-S123, 1996.

HAGLUND, O.; WALLIN, R.; WRETLING, S; HULTBERG, B. \& SALDEEN, T. Effects of fish oil alone and combined with long chain (n-6) fatty acids on 
some coronary risk factors in male subjects. Journal of Nutrition Biochemistry, v. 9, n. 11, p. 629-635, 1998.

HARRIS, W. S.; ASSAD, B. \& POSTON, C. Tissue Omega6/Omega-3 fatty acid ratio and risk for coronary heart disease. Americam Journal Cardioligy, v. 98, n. 4, p. 1926, 2006.

ISO, INTERNATIONAL ORGANIZATION FOR STANDARDIZATION. Animal and vegetable fats and oils preparation of methyl esters of fatty acids. Geneve: ISO. Method ISO 5509, p.1-6, 1978.

LAWRIE, R. A. Ciência da Carne. Trad. JANE MARIA RUBENSAM. 6. ed. - Porto Alegre: Artmed, 2005. 384 p.

LEGOYNIE, C.; BRITZ, T. J. \& HOFFMAN, L. C. Impact of freezing and thawing on the quality of meat: Review. Meat Science, v. 91, p. 93-98, 2012.

MADRUGA, M. S. Artigo Técnico - Carne Caprina: verdades e mitos a luz da ciência. Revista Nacional da Carne, v. 264, n. 23, p. 34-40, 1999.

MADRUGA, M. S. Processamento e características físicas e organolépticas das carnes caprina e ovina. In: IV SEMANA DA CAPRINOCULTURA E OVINOCULTURA BRASILEIRA, p. 1-18. SobralCeará, 2004.

MALAN, S. W. The improved Boer goat. Small Ruminant Research, v. 36, p. 165-170, 2000.

MONTEIRO, A. F. F.; BRAGA, M. E. D. \& MATA, M. E. R. M. C. Congelamento de carne suína a temperaturas criogênicas: alterações de algumas características físicoquímicas. Revista Brasileira de Produtos Agroindustriais, v. 4, n. 1, p. 51-62, 2002.

NIKI, E. Biomarkers of lipid peroxidation in clinical material. Biochimica et Biophysica Acta (BBA) - General Subjects, v. 1840, 809-817, 2014.

PARDI, M. C.; SANTOS, I. F.; SOUZA, E. R. \& PARDI, H. S. Ciência, Higiene e Tecnologia da Carne. $2^{\mathrm{a}}$ ed. Goiânia: CEGRAF-UFG/Niterói: EDUFF, 2006. 624p.

PIETRASIK, Z. \& JANZ J. A. M. Influence of freezing and thawing on the hydration characteristics quality, and consumer acceptance of whole muscle beef injected with solutions of salt and phosphate. Meat Science, v. 81, n. 3, p. 523-532, 2008.

PINKERTON, F. \& McMILLIN, K. Characteristics of goat meat. Meat Goat News, v. 4, n. 6, p. 5-6, 1997.

SAADATIAN-ELAHI, M.; NORAT, T.; GOUDABLE, J. \& RIBOLI, E. Biomarkers of dietary fatty acid intake and the risk of breast cancer: A meta-analysis. International Journal of Cancer, v. 111, p. 584-591, 2004.

SCHROEPFER, T. Factors influencing terminally ill elders' consideration of a hastened death. Annual Scientific Meeting, Gerontological Society of America, 2002.
SIMOPOULOS, A. P. The omega-6/omega-3 fatty acid ratio, genetic variation, and cardiovascular disease. Asia Pacific Journal of Clinic Nutrition, v. 17, n. Suppl 1, p. 131-134, 2008.

SINNHUBER, R. O. \& YU, T. C. 2-Thiobarbituric acid method for measurement of rancidity in fishery products II. The quantitative determination of malonaldehyde. Food Technology, v. 12, p. 9-12, 1958.

SPICKETT, C. M. The lipid peroxidation product 4-hydroxy2-nonenal: Advances in chemistry and analysis. Redox Biology, v.1, p. 145-152, 2013.

STIKA, J. F.; XIONG, Y. L.; SUMAN, S. P.; BLANCHARD, S. P. \& MOODY, W. G. Frozen storage stability of antioxidant-treated raw restructured beef steaks made from mature cows. Meat Science, v. 77, p. $1-8,2007$.

STOLL, B. A. n-3 Fatty acids and lipid peroxidation in breast cancer inhibition British Journal of Nutrition v. 87, p. 193-198, 2002.

TARLADGIS, B. G.; WATTS, B. M. \& YOUNATHAN, M. T. A distillation method for the quantitative determination of malonaldehyde in rancid foods. The Journal of the American Oil Chemist's Society, v. 37, n. 1, p. 44-48, 1960.

YU, M.; GAO, Q.; WANG, Y.; ZHANG, W.; LI, L.; WANG, Y. \& DAI, Y. Unbalanced omega-6/omega-3 ratio in red meat products in China. The Journal of Biomedical Research, v. 27, n. 5, p. 366-371, 2013.

WEBB, E. C.; CASEY, N. H. \& SIMELA, L. Goat meat quality. Small Ruminant Research. v. 60, p.153-166, 2005.

ZAPATA, J. F. F.; NOGUEIRA, C. M.; SEABRA, L. M. J.; BEZERRA, L. C. N. M. \& BESERRA, F. J. Características da carne de pequenos ruminantes do Nordeste do Brasil. Boletim da Sociedade Brasileira de Ciência e Tecnologia de Alimentos, Campinas, v. 37, n. 2, p. 146-153, 2003. 